\title{
Pathogenesis and clinical features of chronic pulmonary aspergillosis -Is it possible to distinguish CNPA and CCPA clinically?-
}

\author{
Koichi Izumikawa ${ }^{1}$, Takayoshi Tashiro ${ }^{2}$, Masato Tashiro ${ }^{1}$, Takahiro \\ Takazono $^{1}$, Kosuke Kosai ${ }^{1}$, Yoshitomo Morinaga ${ }^{3}$, Shintaro Kurihara ${ }^{1}$, Shigeki \\ Nakamura $^{1}$, Yoshifumi Imamura ${ }^{1}$, Taiga Miyazaki ${ }^{1}$, Misuzu Tsukamoto ${ }^{1}$, Hiroshi \\ Kakeya $^{1}$, Tomayoshi Hayashi ${ }^{4}$, Katsunori Yanagihara ${ }^{3}$, Takeshi Nagayasu ${ }^{5}$ and Shigeru \\ Kohno $^{1}$ \\ ${ }^{1}$ Department of Molecular Microbiology and Immunology, ${ }^{2}$ Department of Health \\ Sciences, ${ }^{3}$ Department of Laboratory Medicine, ${ }^{4}$ Department of Pathology, and ${ }^{5}$ \\ Department of Translational Medical Science, Nagasaki University Graduate School of \\ Biomedical Sciences, Nagasaki, Japan
}

Running Title: Case series of CPA and new proposal

Key words: chronic necrotizing pulmonary aspergillosis, chronic cavitary pulmonary aspergillosis, chronic progressive pulmonary aspergillosis 


\section{Corresponding author:}

Koichi Izumikawa, M.D., Ph.D.

Department of Molecular Microbiology and Immunology

Nagasaki University Graduate School of Biomedical Sciences

1-7-1 Sakamoto

Nagasaki 852-8501, JAPAN

Phone: +81-95-819-7273

Fax: +81-95-849-7285

E-mail: koizumik@nagasaki-u.ac.jp 


\section{SUMMARY (198 words)}

\section{Background}

The pathogenesis of chronic pulmonary aspergillosis (CPA) including chronic necrotizing pulmonary aspergillosis (CNPA), chronic cavitary pulmonary aspergillosis (CCPA), and simple aspergilloma (SA) has been poorly investigated. We examined all types of CPA cases with histopathological evidence to clarify the differences in pathogenesis and clinical features.

\section{Method}

We searched for cases diagnosed as pulmonary aspergillosis by histopathological examination in Nagasaki University Hospital between 1964 and September 2010. All available clinical information including radiological findings were collected and analyzed.

\section{Result}

We found 7, 5, 8, and 7 cases of proven CNPA, probable CNPA, CCPA, and SA, respectively. The radiograph of proven and probable CNPA was initially infiltrates or nodules that progress to form cavities with or without aspergilloma, whereas the radiograph of CCPA showed pre-existed cavities and peri-cavitary infiltrates with or without aspergilloma. The patients with proven and probable CNPA exhibited not only respiratory symptoms but also systemic symptoms and malnutrition. Aspergillus fumigatus was the most frequently isolated Aspergillus species $(\mathrm{n}=14)$, however, $A$. niger was the predominant isolated species in proven CNPA cases $(n=4)$.

\section{Conclusion}

Our data indicate that the cases with chronic infiltration, progressive cavitation, and subsequent aspergilloma formation should be diagnosed as CNPA, and the cases with 
pre-existed cavities showing peri-cavitary infiltrates with or without aspergilloma would mean CCPA. However, it may be difficult to distinguish the two subtypes if a series of adequate radiography films are not available. We propose the term "chronic progressive pulmonary aspergillosis (CPPA)” for the clinical syndrome including both CNPA and CCPA. 


\section{INTRODUCTION}

Chronic pulmonary aspergillosis (CPA) was originally established in the early 1980s by Binder as chronic necrotizing pulmonary aspergillosis (CNPA) [1] and semi-invasive aspergillosis (SIA) by Gefter [2]. CNPA/SIA is characterized by a slow progressive cavitating process in the lungs due to Aspergillus spp. infection. In the last decade, new clinical nomenclature and definition of chronic forms of aspergillosis have been proposed [3-5], and recent guidelines from the Infectious Diseases Society of America (IDSA) have indicated 3 major subtypes of chronic forms of pulmonary aspergillosis, namely CNPA (categorized in subacute invasive form of aspergillosis; subacute IPA), chronic cavitary pulmonary aspergillosis (CCPA), and aspergilloma [6]. Aspergilloma was traditionally classified as simple or complex in the surgical literature, and complex aspergilloma is considered CCPA by current IDSA guidelines [6, 7]. Updated IDSA guidelines and textbook have indicated that the differences between CNPA and CCPA include prolonged time frame (CNPA, 1-3 months vs. CCPA, >3 months) [8]. The original CNPA cases defined by Binder are not equal to those of CNPA cases defined by Denning. Establishing the precise sub-classifications of CPA, while challenging, is important for clinical trials as well as the development of tools for its diagnosis and treatment. The pathogenesis of each type of CPA examined by pathological samples has been poorly investigated. In this study, we investigated all types of CPA cases including CNPA, CCPA, and simple aspergilloma (SA) diagnosed by histopathological examination to clarify the differences in the pathogenesis and clinical features of each type of CPA. In particular, revealing the difference between CNPA and CCPA is the most important of goal of our study. This study was performed in a single medical teaching hospital in Japan. 


\section{MATERIAL AND METHODS}

\section{Case collection and analysis}

We searched for cases diagnosed as “pulmonary aspergillosis” by histopathological examination of respiratory specimens acquired by biopsy, surgical resection, or autopsy in Nagasaki University Hospital. Nagasaki University Hospital is a Japanese teaching hospital that contains 860 beds and is located in the southwestern part of Japan. All cases registered in the database between 1964 and September 2010 were screened, and patient data such as sex, age, underlying diseases, clinical symptoms, clinical course, laboratory and radiological findings, and treatment was collected from medical records and analyzed. This retrospective study was approved by the ethics committee of Nagasaki University Hospital.

\section{Definition of pulmonary aspergillosis subtypes by histopathological and clinical aspects}

The histopathological and clinical definition of invasive pulmonary aspergillosis (IPA), CNPA, CCPA, and SA in this study is indicated in Figure 1. A diagnosis of IPA and CNPA requires extensive hyphal invasion in the lung parenchyma and localized hyphal invasion in cavity walls and/or destroyed lung tissue, respectively. Non-invasive CPA was subdivided into three groups as Group A, CCPA and SA. CCPA requires preexisting cavities and disease progression within 3 months. SA requires a solitary cavitary legion with an aspergilloma and no disease progression within 3 months. Group A does not match to either of CNPA and CCPA. This group requires a newly developed necrotic lung cavity and no hyphal invasion in any lung tissue. The existence of preexisting cavity was confirmed by X-ray films taken prior to the disease onset. 


\section{Statistical analysis}

The chi-square test was used for qualitative variables. Multivariate logistic regression analysis was performed using the variables that were selected from univariate analysis $(P<0.1)$. This analysis was performed by SPSS ver. 16 software. $P$ values $<0.05$ were considered statistically significant. 


\section{RESULTS}

\section{Characters of CPA cases and patients}

A total of 60 cases of which histopathological findings indicated pulmonary aspergillosis were found in the database. Then the cases of IPA and allergic bronchopulmonary aspergillosis (ABPA) were excluded. We searched the patients' medical records including X-ray films and accepted 27 cases of CPA for further analysis due to their availability of medical information and X-ray films. We found 7, 5, 8, and 7 cases of CNPA, Group A, CCPA, and SA, respectively. They were histopathologically diagnosed by surgical resection in 19, biopsy in 4, and autopsy in 4 cases, respectively.

Table 1 shows sex, age, body mass index, symptoms, and disease duration prior to diagnosis. The cases of CNPA, Group A and CCPA demonstrated a male predominance, and the body mass index values of patients with CNPA and Group A were statistically lower than those of patients with SA. Several patients with SA complained of cough, hemoptysis, and sputum; however, the symptoms or radiographic findings did not indicate disease progression within 3 months. All cases with CNPA, Group A and CCPA complained respiratory symptoms. Progressive fever and weight loss were found in only CNPA and Group A cases, which may reflect progressive tissue destruction and more direct inflammatory responses than CCPA and SA does.

The underlying conditions of patients with CPA are indicated in Table 2. Patients with CNPA most frequently had chronic obstructive pulmonary disease (COPD) or emphysema, whereas patients with CCPA and SA most frequently had prior tuberculosis. A history of pneumonia was detected in 3/7 and 4/5 cases of CNPA and Group A, respectively, and may have been caused by a direct or indirect response to Aspergillus spp. A history of thoracic surgery or pneumothorax was also detected in 
CNPA and Group A cases. Diabetes mellitus was the predominant underlying systemic disease in all subtypes of CPA cases. Cigarette smoking was also seen in all subtypes of CPA cases. Malnutrition was seen in 4/7, 4/5 and 3/8 cases of Group A, CNPA and CCPA, respectively.

\section{Radiological findings of patients with CPA}

Typical radiographic images of each subtypes of CPA are presented in Figure 2. The radiograph of CNPA and Group A cases was initially infiltrates that progress to form cavities with or without aspergilloma. The radiograph of CCPA cases showed preexisted cavities and peri-cavitary infiltrates with or without aspergilloma, and SA cases showed a single cavity with aspergilloma. Radiological findings at the time of diagnosis are summarized in Table 3. The upper lobes were affected in all cases of CPA, while both the right and left upper lobes were affected in 4/7 and 1/5 cases of CNPA and Group A, respectively. Although the upper lobes were most commonly affected, the middle and the lower lobes were also affected in CCPA and SA cases. Cavitary infiltrates with aspergilloma was the predominant finding in CNPA and Group A cases. Cavitary nodules with aspergilloma were also recognized in CNPA cases. Cavities with aspergilloma were most commonly found in CCPA cases. All SA cases showed a single cavity with aspergilloma. Aspergillomas were recognized in 4/7, 5/5, 6/8, and 7/7 cases of CNPA, Group A, CCPA, and SA, respectively.

\section{Laboratory findings of patients with CPA}

Laboratory findings are presented in Table 4. Aspergillus spp. were isolated in 6/7 (86\%), 4/5 (80\%), 7/8 (88\%), and 4/7 (57\%) from CNPA, Group A, CCPA, and SA 
cases, respectively. A. fumigatus was the most frequently isolated Aspergillus species (n = 14), followed by A. niger $(n=5)$, A. flavus $(n=2)$, and A. terreus $(n=1)$ in all CPA cases; while A. niger was the predominant isolated species in CNPA cases $(n=4)$. AntiAspergillus antibody tests were positive in 3/5 (60\%), 3/4 (75\%), 4/8 (50\%), and 1/6 (17\%) cases of CNPA, Group A, CCPA, and SA, respectively. Aspergillus antigen tests were positive in 5/6 (83\%), 4/5 (80\%) and 6/6 (100\%) cases of CNPA, Group A and CCPA, respectively. However, it was negative in all SA cases. A few CPA cases had a positive $\beta$-D-glucan test. The inflammatory marker results indicated a lower positive rate of leukocytosis compared to C-reactive protein and erythrocyte sedimentation rate (ESR), although the number of cases in which ESR was measured was low. The number of cases in which any of the 3 inflammatory markers tested positive was 5/7 (71\%), 5/5 (100\%), 4/8 (50\%), and 2/7 (29\%) in CNPA, Group A, CCPA, and SA, respectively.

\section{DISCUSSION}

The pathogenesis of CPA, especially CNPA and CCPA, has not been widely investigated, and the noncommittal definition criteria and overlapping clinical features make it difficult to distinguish the two subtypes. The clinical time course, radiological findings, and defects in innate immunity may be indexes of differences as suggested by Denning and others [3, 5, 6, 8, 9], however, sub-classification of CPA should be made according to the pathogenesis of the disease on the basis of pathological and radiological features. Although this study is retrospective, we collected a total of 27 CPA cases with complete pathological, radiological, microbiological, and serological findings.

The clinical distinction between SA and other CPA subtypes may not be difficult 
because of it presents with obvious radiological findings and clinical features. All 7 SA cases in our series showed a solitary cavity with an aspergilloma. On the other hand, discerning the difference between CNPA and CCPA is challenging. Binder et al. described that CNPA is defined as an indolent, cavitating process in the lungs due to the invasion of lung tissue by a fungus of the Aspergillus spp.. Gefter et al. reported that the radiographic features of SIA include chronic infiltration, progressive cavitation, and subsequent mycetoma formation, and that the absence of a previous cavity distinguishes such cases from secondary noninvasive mycetomas. While, Denning et al. defined CNPA as subacute invasive form of aspergillosis, occurring over 1-3 month, and CCPA as non-invasive chronic cavitary aspergillosis, occurring $>3$ months. According to Denning et al., the development of CCPA lesions involves two possible ways of cavity formation: 1) the infiltrates were initially ill-defined areas of consolidation that progressed to form well-defined cavit

es; and 2) the cavities were pre-existing (i.e., in cases of previous tuberculosis or bronchiectasis) [4]. However, the former radiographic features are concordant with those of Binder's CNPA or Gefter's SIA. Thus, the consensus of definition of CNPA is not established, and some cases of Denning’s CCPA may be Binder’s CNPA.

In this study, we defined the cases with progressive cavitating processes in the lung without a pre-existing cavity and presence of hyphal invasion into lung parenchyma as CNPA, and the cases with pre-existing cavities and peri-cavitary infiltrates and presence of hyphae in a cavity but not in lung parenchyma as CCPA. However, whether group A in our series is classified into CNPA or CCPA is controversial. The radiographic features of CNPA and Group A cases are similar; they are chronic infiltration, progressive cavitation and subsequent aspergilloma formation. The pathological features of the 
cavity wall of CNPA and Group A cases are similar except hyphal invasion; they are acute and chronic inflammation, granulation, parenchymal necrosis and subsequent cavity formation with or without fragments of destroyed lung tissue in the cavity. The hyphal invasion into cavity wall was observed only in CNPA cases but not Group A cases. Antifungals treatment before the pathological examinations were conducted in 3/7 and 4/5 cases of CNPA and Group A, respectively. Additionally, duration of treatment was relatively longer in Group A cases (6 weeks to 8 months) compared to that of CNPA(10 days for two case) except one case (approximately 14 months). It is possible that antifungal treatment prior to the pathological examination may block the hyphal invasion of Aspergillus in Group A cases. Moreover, systemic symptoms such as fever and weight loss, and underlying conditions such as history of pneumonia or thoracic surgery are common to both CNPA and Group A, but not to CCPA. Thus, the pathological and clinical features and backgrounds of Group A cases were resemble to those of CNPA cases; which means the pathogenesis of CNPA and Group A cases is considered the same.

It is supposed that Aspergillus infects air spaces, such as emphysematous bullae, then destroys lung tissue by invasion and/or mycotic toxins, proteolytic enzymes, and metabolites produced by Aspergillus. Proteolytic enzymes and oxidant derived from neutrophils and macrophages may also cause tissue necrosis. Thus, the cavity formation can be developed even though the hyphal invasion is minimal or absent. Therefore, the cases with chronic infiltration, progressive cavitation, and subsequent aspergilloma formation should be diagnosed as CNPA; the cases in which hyphal invasion of lung tissue is demonstrated are proven CNPA and the cases in which the invasion is not demonstrated are probable CNPA. Hence, we diagnose the Group A in our series as 
probable CNPA. The median duration of the disease was 3 and 24 months in proven CNPA and probable CNPA (Group A) cases, respectively. As Binder et al. mentioned that the disease is usually of 1 to 6 months duration but can be present for years prior to diagnosis, CNPA is not always subacute (1-3 months).

There were pre-existing cavities such as sequelae of pulmonary tuberculosis, bronchiectasis, COPD, bullae, and interstitial pneumonia in all 8 of CCPA cases, and the wall of these cases was composed of chronic inflammatory, granulation, and fibrous tissue layers, and the inner surface of the cavity is covered by bronchial epithelium or metaplastic squamous cell epithelium in part. Thus, CCPA is synonymous with complex aspergilloma. Our study results indicate that CNPA can be distinguishable from CCPA by careful observation of progression of the cavitary lesion if a series of adequate radiography films are available. In some cases, however, it will be difficult to distinguish the two subtypes because adequate films are not always available.

The limitations of our study include its retrospective nature, the number of cases being insufficient for establishing definite criteria, and the need for additional information. The importance of differentiating between CNPA and CCPA is scientific; however, it may be difficult because of the noncommittal definition criteria and existence of overlapping findings. From the management perspective, its elucidation does not provide deeper insight into the differentiation, except for executing clinical trials. Although we respect and follow the original definition of CNPA and the concept of CCPA, there is a limitation to distinguishing these two entities in actual clinical settings as discussed above. Hope et al. indicated that apparent distinct entities do not exist for the CPA subtypes and that these forms usually overlap; therefore, the requirement and importance of the rigorous sub-classifications of CPA are unclear [5]. 
Hence, we propose the term “chronic progressive pulmonary aspergillosis (CPPA)” for the clinical syndrome including both CNPA and CCPA [10]. Therefore, CPA is consisted of CPPA and SA.

\section{Acknowledgements}

We appreciated Yosuke Nagayoshi, Akitaka Tanaka, Asuka Minematsu, Katsuji Hirano, Naoki Iwanaga, Shotaro Ide, Kazuaki Takeda, and Masataka Yoshida for gathering clinical data.

Financial disclosures: The authors have no potential conflicts of interest to disclose. 


\section{FIGURE LEGENDS}

Figure 1. Histopathological and clinical diagnosis of pulmonary aspergillosis in this study.

A diagnosis of invasive pulmonary aspergillosis and chronic necrotizing pulmonary aspergillosis (CNPA) requires extensive hyphal invasion in the lung parenchyma and localized hyphal invasion in cavity walls and/or destroyed lung tissue, respectively. Group A, chronic cavitary pulmonary aspergillosis (CCPA), and simple aspergilloma (SA) require no hyphal invasion in any lung tissue; however, Group A requires new cavity formation and clinical deterioration without a pre-existing cavitary lesion in the lung. CCPA requires pre-existing cavities and disease progression and SA requires a solitary cavitary legion with an aspergilloma and no clinical deterioration within 3 months.

Figure 2. Chest radiographic images of CPA cases

A) CNPA: the patient (75-year-old man) had chronic obstructive lung disease and a history of left pneumonectomy for intractable pulmonary tuberculosis and aspergilloma 7 years before admission. The radiographs show consolidation around bullae and pleural thickening in the right upper lung. B) Group A: the patient (75-year-old man) had diabetes mellitus and a history of pneumonia 2 years before admission. The radiographs show consolidation around cavities with aspergilloma and pleural thickening in the right upper lung. C) CCPA: the patient (69-year-old man) had a history of pulmonary 
tuberculosis. The radiographs show multiple cavities with aspergilloma in the right upper lung. D) Simple aspergilloma: the patient (40-year-old man) had a history of bullectomy for left pneumothorax 6 years before admission. The radiographs show a single cavity with an aspergilloma in the left upper lung. 
Table 1. Characteristics of CPA patients

\begin{tabular}{lcccc}
\hline & CNPA $(\mathrm{n}=7)$ & Group A $(\mathrm{n}=5)$ & CCPA $(\mathrm{n}=8)$ & SA $(\mathrm{n}=7)$ \\
\hline Sex; Male/Female & $6 / 1$ & $4 / 1$ & $7 / 1$ & $3 / 4$ \\
Age; mean (range) & $59.1(45-75)$ & $67.0(50-77)$ & $69.7(58-80)$ & $56.6(40-68)$ \\
Body mass index; mean (range) & $18.0 *(13.0-21.4)$ & $16.9 *(13.9-18.8)$ & $20.2(15.7-25.7)$ & $21.3(18.0-23.9)$ \\
Symptoms & & & & \\
cough & 5 & 5 & 7 & 4 \\
sputum & 5 & 2 & 3 & 3 \\
hemoptysis & 1 & 5 & 6 & 4 \\
dyspnea & 3 & 1 & 2 & 0 \\
fever & 4 & 2 & 0 & 0 \\
malaise & 3 & 0 & 0 & 0 \\
weight loss & 1 & 2 & 0 & 0 \\
Duration; median (range) & $3 \mathrm{M}(1 \mathrm{~m}-6 \mathrm{y})$ & $24 \mathrm{M}(17 \mathrm{~m}-6 \mathrm{y})$ & $29 \mathrm{M}(6 \mathrm{~m}-5 \mathrm{y} 4 \mathrm{~m})$ & $24 \mathrm{M}(6 \mathrm{~m}-4 \mathrm{y})$ \\
\hline
\end{tabular}

CNPA, chronic necrotizing pulmonary aspergillosis; CCPA, chronic cavitary pulmonary aspergillosis; SA, simple pulmonary aspergilloma; m,

months; and y, years.

*: statistical difference between CNPA, Group A and SA was indicated $(\mathrm{p}<0.05)$ 
Table 2. Underlying conditions of CPA patients

\begin{tabular}{|c|c|c|c|c|}
\hline & CNPA $(n=7)$ & Group A $(n=5)$ & $\mathrm{CCPA}(\mathrm{n}=8)$ & $\mathrm{SA}(\mathrm{n}=7)$ \\
\hline \multicolumn{5}{|l|}{ Respiratory conditions } \\
\hline Prior tuberculosis & 2 & 1 & 3 & 4 \\
\hline Bronchiectasis & 1 & 3 & 2 & 3 \\
\hline Prior pneumonia & 3 & 4 & 0 & 2 \\
\hline Pneumothorax or bullae & 1 & 3 & 2 & 2 \\
\hline COPD or emphysema & 5 & 0 & 1 & 0 \\
\hline Prior thoracic surgery & 2 & 2 & 1 & 1 \\
\hline Others respiratory conditions* & 2 & 4 & 9 & 4 \\
\hline \multicolumn{5}{|l|}{ Systemic conditions } \\
\hline Diabetes Mellitus & 3 & 2 & 2 & 2 \\
\hline Steroid usage & 2 & 0 & 2 & 0 \\
\hline Other systemic conditions** & 4 & 0 & 3 & 8 \\
\hline Smoking (>20y) & 5 & 2 & 7 & 2 \\
\hline Malnutrition $(\mathrm{BMI}<18.5)$ & 4 & 4 & 3 & 1 \\
\hline
\end{tabular}


CNPA, chronic necrotizing pulmonary aspergillosis; CCPA, chronic cavitary pulmonary aspergillosis; SA, simple pulmonary aspergilloma; COPD, chronic obstructive pulmonary disease

*: bronchial asthma (4 cases), lung cancer (4 cases), chronic parasinusitis (4 cases), prior pleuritis (2 cases), interstitial pneumonia (2 cases), eosinophilic pneumonia (1 case), non-tuberculous mycobacteriosis (1 case) and pneumoconiosis (1 case)

**: gastric cancer (2 cases), colon cancer (1 case), prostate cancer (1 case), rheumatoid arthritis (1 case), Harada disease (1 case), ovarial cyst (2 cases), nasal disease (3 cases), liver disease (1 case), alcoholism (2 cases), and acoustic neuroma (1 case). 
Table 3. Radiological findings of CPA patients

\begin{tabular}{|c|c|c|c|c|}
\hline & CNPA $(n=7)$ & Group A $(n=5)$ & CСРА $(n=8)$ & $\mathrm{SA}(n=7)$ \\
\hline \multicolumn{5}{|l|}{ Affected sites } \\
\hline RUL + LUL & 4 & 1 & 0 & 0 \\
\hline RUL & 3 & 3 & 3 & 2 \\
\hline RML & 0 & 0 & 2 & 2 \\
\hline RLL & 0 & 0 & 1 & 1 \\
\hline LUL & 0 & 1 & 0 & 2 \\
\hline LLL & 0 & 0 & 2 & 0 \\
\hline Cavities with aspergilloma & 2 & 1 & 5 & 0 \\
\hline Cavity with aspergilloma & 1 & 0 & 1 & 7 \\
\hline Cavities without aspergilloma & 1 & 1 & 0 & 0 \\
\hline Cavitary infiltrates with aspergilloma & 3 & 4 & 0 & 0 \\
\hline Cavitary infiltrates without aspergilloma & 1 & 0 & 0 & 0 \\
\hline Cavitary nodules with aspergilloma & 2 & 0 & 0 & 0 \\
\hline Multiple nodules & 1 & 0 & 0 & 0 \\
\hline Bronchiectasis and infiltrate* & 0 & 0 & 1 & 0 \\
\hline Bronchiectasis and atelectasis* & 0 & 0 & 1 & 0 \\
\hline
\end{tabular}


Table 4. Laboratory findings of CPA patients

\begin{tabular}{|c|c|c|c|c|}
\hline & CNPA $(n=7)$ & Group A (n=5) & $\operatorname{CCPA~}(n=8)$ & $\mathrm{SA}(\mathrm{n}=7)$ \\
\hline \multicolumn{5}{|l|}{ Isolation of Aspergillus spp. } \\
\hline A. fumigatus & 2 & 4 & 5 & 3 \\
\hline A. niger & 4 & 0 & 1 & 0 \\
\hline A. flavus & 0 & 0 & 1 & 1 \\
\hline A. terreus & $1^{*}$ & 0 & 0 & 0 \\
\hline not identified & 1 & 1 & 1 & 3 \\
\hline \multicolumn{5}{|l|}{ Serological findings } \\
\hline Anti-aspergillus antibody $(+)$ & $3 / 5$ & $3 / 4$ & $4 / 8$ & $1 / 6$ \\
\hline Aspergillus antigen ( $\geq 0.5$ C.O.I.) & $5 / 6$ & $4 / 5$ & $6 / 6$ & $0 / 5$ \\
\hline$\beta$-D-glucan $(\geq 20 \mathrm{pg} / \mathrm{ml})$ & $2 / 6$ & $1 / 4$ & $1 / 5$ & $1 / 4$ \\
\hline \multicolumn{5}{|l|}{ Inflammatory makers } \\
\hline Leukocytosis (WBC $>9000 / \mathrm{mm}^{3}$ ) & 2 & 0 & 1 & 0 \\
\hline $\mathrm{CRP}(>0.3 \mathrm{mg} / \mathrm{dl})$ & 5 & 4 & 4 & 0 \\
\hline ESR $(>20$ mm/h) & $1 / 1$ & $2 / 2$ & $3 / 3$ & $2 / 4$ \\
\hline
\end{tabular}

CNPA, chronic necrotizing pulmonary aspergillosis; CCPA, chronic cavitary pulmonary aspergillosis; SA, simple pulmonary aspergilloma; CRP,

C-reactive protein; and ESR, Erythrocyte sedimentation rate.

*: A. terreus was isolated with A. fumigatus from the same patient 


\section{REFERENCES}

1. Binder RE, Faling LJ, Pugatch RD, Mahasaen C, Snider GL. Chronic necrotizing pulmonary aspergillosis: a discrete clinical entity. Medicine (Baltimore). 1982;61:10924.

2. Gefter WB, Weingrad TR, Epstein DM, Ochs RH, Miller WT. "Semi-invasive" pulmonary aspergillosis: a new look at the spectrum of aspergillus infections of the lung. Radiology. 1981;140:313-21.

3. Denning DW. Chronic forms of pulmonary aspergillosis. Clin Microbiol Infect. 2001;7 Suppl 2:25-31.

4. Denning DW, Riniotis K, Dobrashian R, Sambatakou H. Chronic cavitary and fibrosing pulmonary and pleural aspergillosis: case series, proposed nomenclature change, and review. Clin Infect Dis. 2003;37 Suppl 3:S265-80.

5. Hope WW, Walsh TJ, Denning DW. The invasive and saprophytic syndromes due to Aspergillus spp. Med Mycol. 2005;43 Suppl 1:S207-38.

6. Walsh TJ, Anaissie EJ, Denning DW, et al. Treatment of aspergillosis: clinical practice guidelines of the Infectious Diseases Society of America. Clin Infect Dis. 2008;46:32760.

7. Riscili BP, Wood KL. Noninvasive pulmonary Aspergillus infections. Clin Chest Med. 2009;30:315-35, vii.

8. Denning DW. Aspergillosis. 17th ed. New York, U.S.A.: McGraw-Hill Companies; 
2008.

9. Smith NL, Denning DW. Underlying conditions in chronic pulmonary aspergillosis including simple aspergilloma. Eur Resp J. 2011;37:865-72.

10. Tashiro T, Izumikawa K, Tashiro M, et al. Case series of chronic necrotizing pulmonary aspergillosis and new proposal. J J Infect Dis. 2013;66:312-316. 
Figure 1. Histopathological and clinical diagnosis of pulmonary aspergillosis in this study

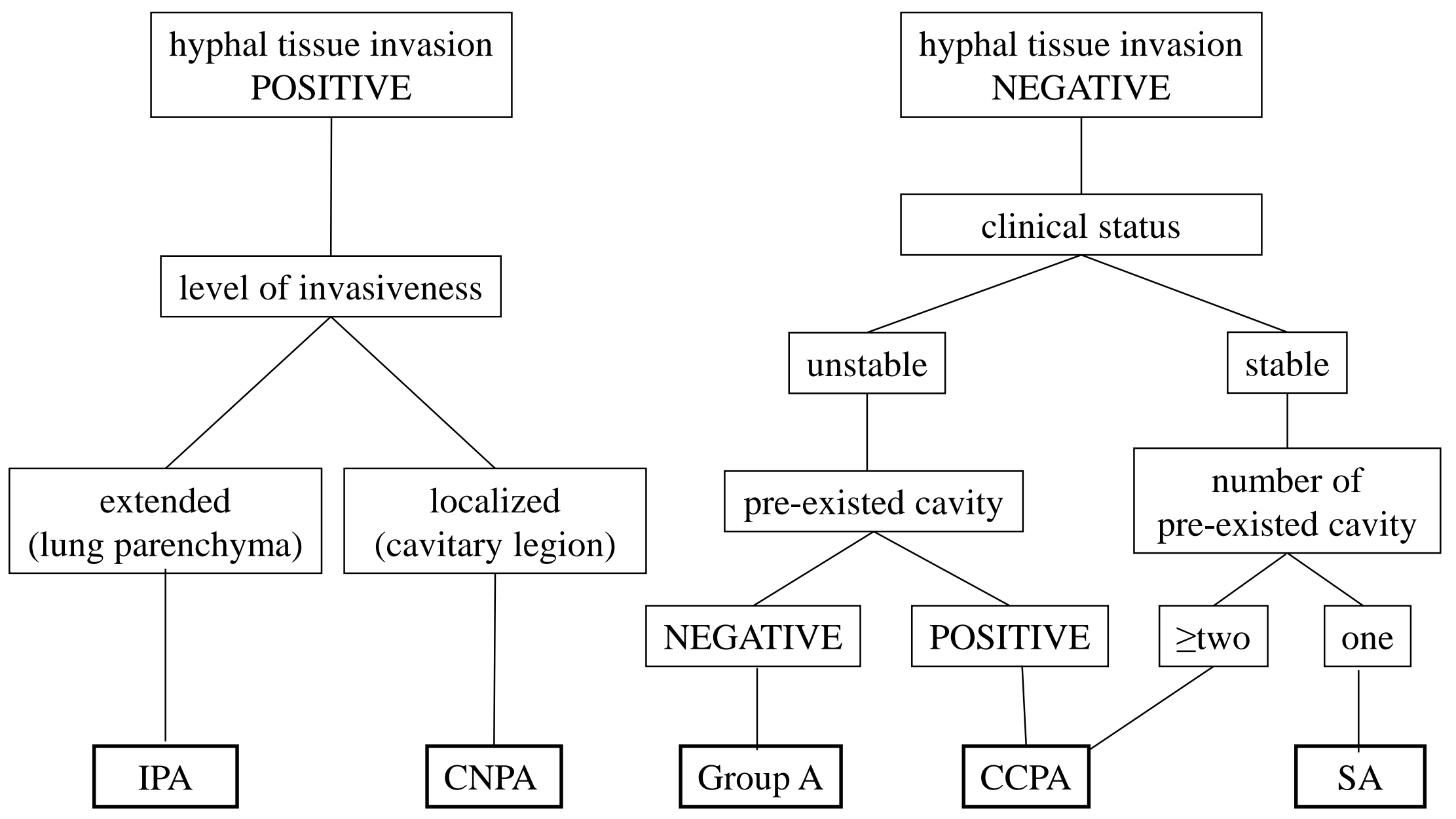


Figure 2. Chest radiographic images of CPA cases

A)

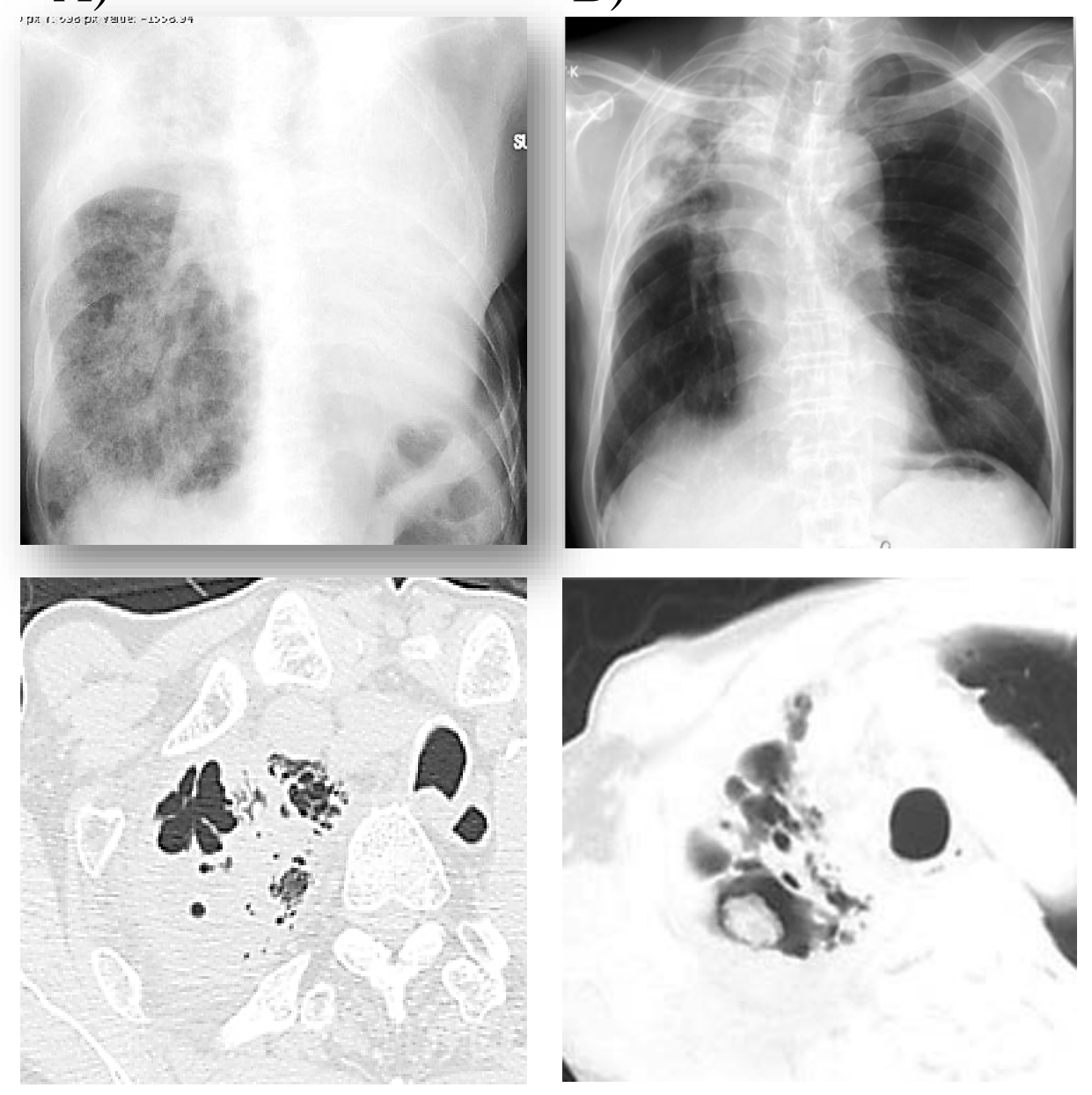

C)
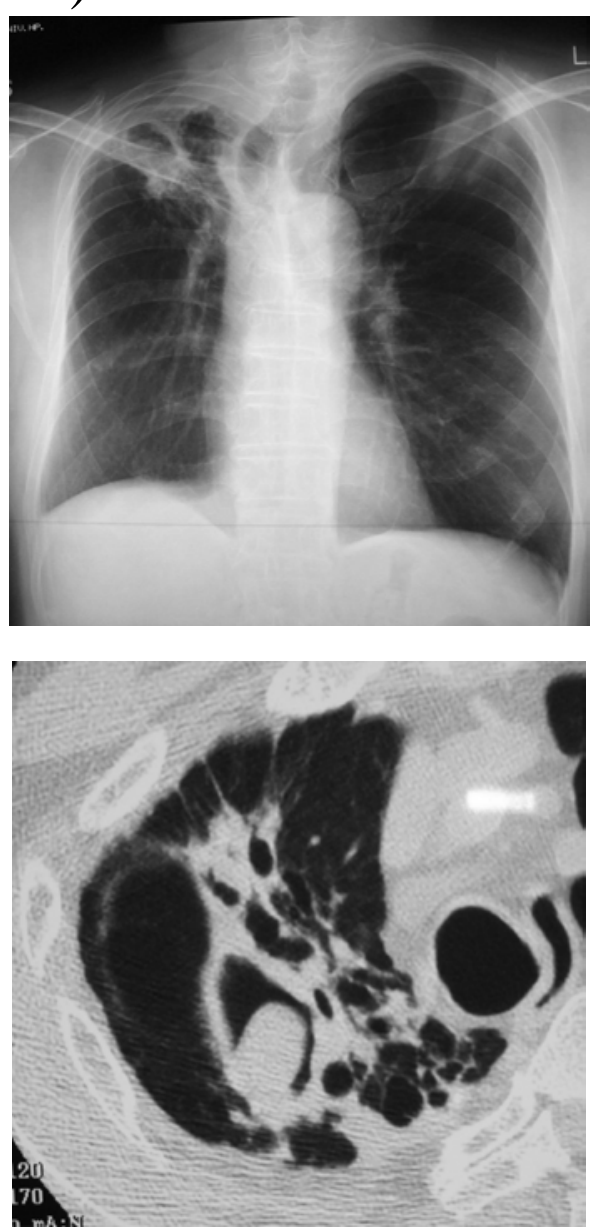

D)
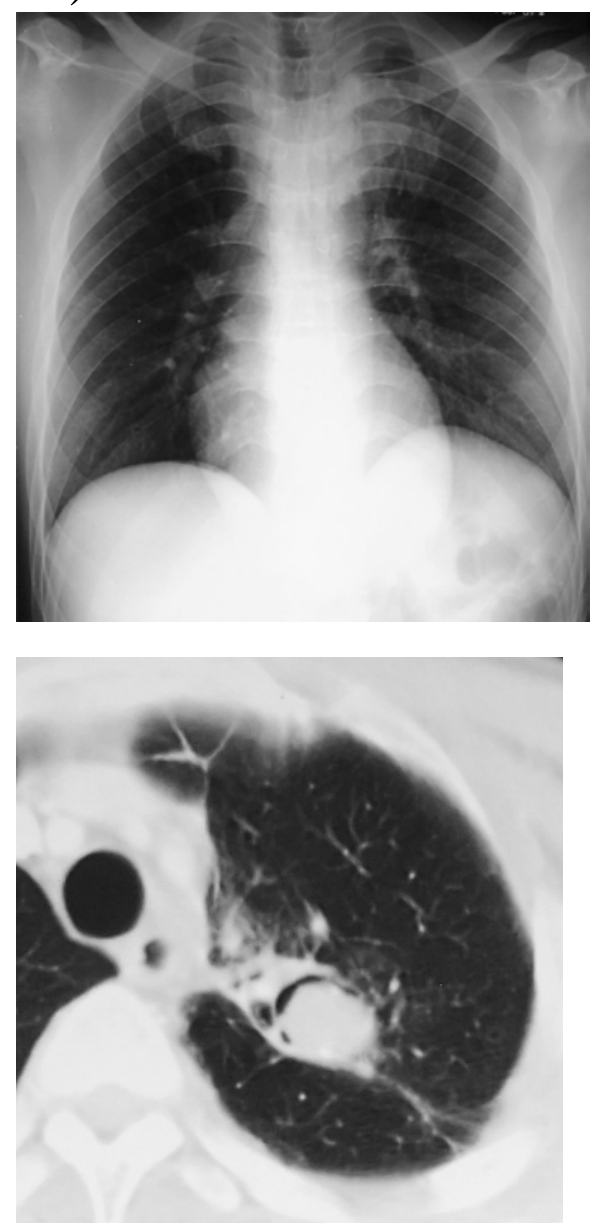